\title{
Sum-Rate Capacity Evaluation Methodology for MIMO-OFDMA Systems
}

\author{
Han-Shin Jo \\ Dept. of Electronics \& Control Engineering, Hanbat National University, Daejeon, \\ Korea \\ hsjo@hanbat.ac.kr
}

\begin{abstract}
Recently, the long-term evolution (LTE) system has emerged as a major concern within the 3rd Generation Partnership Project (3GPP and 3GPP2). Orthogonal frequency division multiple accesses (OFDMA) have been considered within the LTE system as a major technology for cellular radio access. In addition, numerous research groups have proposed the incorporation of technologies such as multi-user scheduling, multi-input multi-output (MIMO), and adaptive modulation and coding (AMC) into OFDMA. However, system-level simulation is required to assess how these technologies interact and to evaluate and optimize each technology with respect to system-level performance in real cellular environments. This article discusses an evaluation methodology of system-level simulation for a MIMO-OFDMA system. The approach by which the capacity evaluation of the MIMO-OFDMA system was conducted and resulting sum-rate capacity is discussed.
\end{abstract}

Keywords: Multicarrier spatial channel model, OFDMA, MIMIO, space-division multiple access (SDMA), system-level simulation

\section{Introduction}

Recently, the 3rd Generation Partnership Project (3GPP and 3GPP2) future long-term evolution (LTE) workshop has opened. The main goal of this project is to obtain a standard that supports demanded variety services and increases competitiveness for dealing with sudden changes in the wireless communication market. In this workshop, the requirements of the LTE system in the physical layer were determined as follows. The system should operate on a broadband, provide high spectral efficiency, support scalable bandwidth and high-speed data services, and support simple transmitter and receiver structure. Orthogonal frequency division multiple accesses (OFDMA) have been considered within the LTE system as a major technology for cellular radio access [1]. In addition, many research groups have proposed the incorporation of technologies such as multi-user scheduling, multi-input multi-output (MIMO), and adaptive modulation and coding (AMC) into OFDMA. The 3GPP will discuss the relative strengths and weaknesses of the proposed technologies through both link-level and system-level simulation. In particular, a system-level simulation is required to assess how these technologies interact and to evaluate and optimize each technology with respect to system performance in real cellular environments.

In this article, we discuss an evaluation methodology of sum-rate capacity achieved by a MIMO-OFDMA system. The methodology focuses on the capacity evaluation of a downlink of a cellular system in multi-cell and multi-user environments. We extend the spatial channel model (SCM) proposed for a single carrier MIMO system to that for the MIMO-OFDMA 
system. Multi-user scheduling schemes, which adaptively assign the frequency and space resources of the MIMO-OFDMA system to mobile stations (MSs), are considered and compared with respect to the system-level capacity. The equivalent signal to noise ratio (SNR) model (ESM) is discussed for predicting the link-level performance in the systemlevel simulation. Finally, we present the system-level evaluation results and discuss how these technologies interact.

\section{MIMO-OFDMA System Overview}

OFDMA is an emerging multiple access technology for future wireless communications. It is based on orthogonal frequency division multiplexing (OFDM) and inherits OFDM's immunity to inter-symbol interference and frequency selective fading. Furthermore, OFDMA provides much higher flexibility in terms of utilizing frequency resources compared with OFDM based approaches. Achieving high transmission rates depends on the ability to provide efficient and flexible resource allocation. This becomes feasible by incorporating multi-user scheduling into OFDMA. In multi-user environments, OFDMA can take advantage of multi-user diversity by twodimensional scheduling, i.e., scheduling the MSs that experience better channel conditions per sub-band (a set of adjacent sub-carriers within coherence bandwidth) and time slot.

MIMO, which is also considered as an important technology of future wireless systems, is particularly well suited for OFDMA because the narrow band OFDMA signal removes complex channel equalization schemes in MIMO reception. Furthermore, combined with OFDMA, MIMO technology can further extend the dimension of scheduling to the space domain via space division multiple access (SDMA) [2-4], where multiple data streams are simultaneously transmitted to the MSs with better channel conditions in the space domain. The synergistic effects realized through the combination of OFDMA with MIMO have led to widespread recognition of MIMO-OFDMA as a potential candidate for future wireless access technologies. Several wireless networking (e.g., IEEE 802.16 and IEEE 802.20) have already been developed using MIMO-OFDMA.

\section{Evaluation Methodology}

A sound evaluation methodology is required for various technologies to be properly evaluated and fairly compared to each other. The methodology should include details on the cellular model, channel model, procedure of simulation, and how to predict link-level performance in a system-level simulation. The simulation assumptions and procedures in this article follow the evaluation methodology described in this section.

The simulation procedure for evaluating a MIMO-OFDMA system is described in Figure 1. The whole simulation is conducted over a certain number of drops. Each drop consists of a large number of OFDM frames, where each is composed of a certain number of OFDM symbols. For each drop a radio network is configured based on the desired system parameters and MSs are uniformly distributed over the radio network, which remains unchanged over a drop. For each OFDM frame, the channel coefficients are generated by the multi-carrier spatial channel model for all sector-MS pairs. Each MS then measures the channel quality for both the sub-band and the transmit antenna and reports these values to its serving sector. The packet scheduler at each sector decides the MSs to be scheduled for data transmission. The transmission rate of the scheduled MS is decided by the AMC scheme. The ESM method then 
allows to compute the packet error rate (PER) and determines whether to ask for a retransmission. This process is repeated until the desired number of OFDM frames is reached.

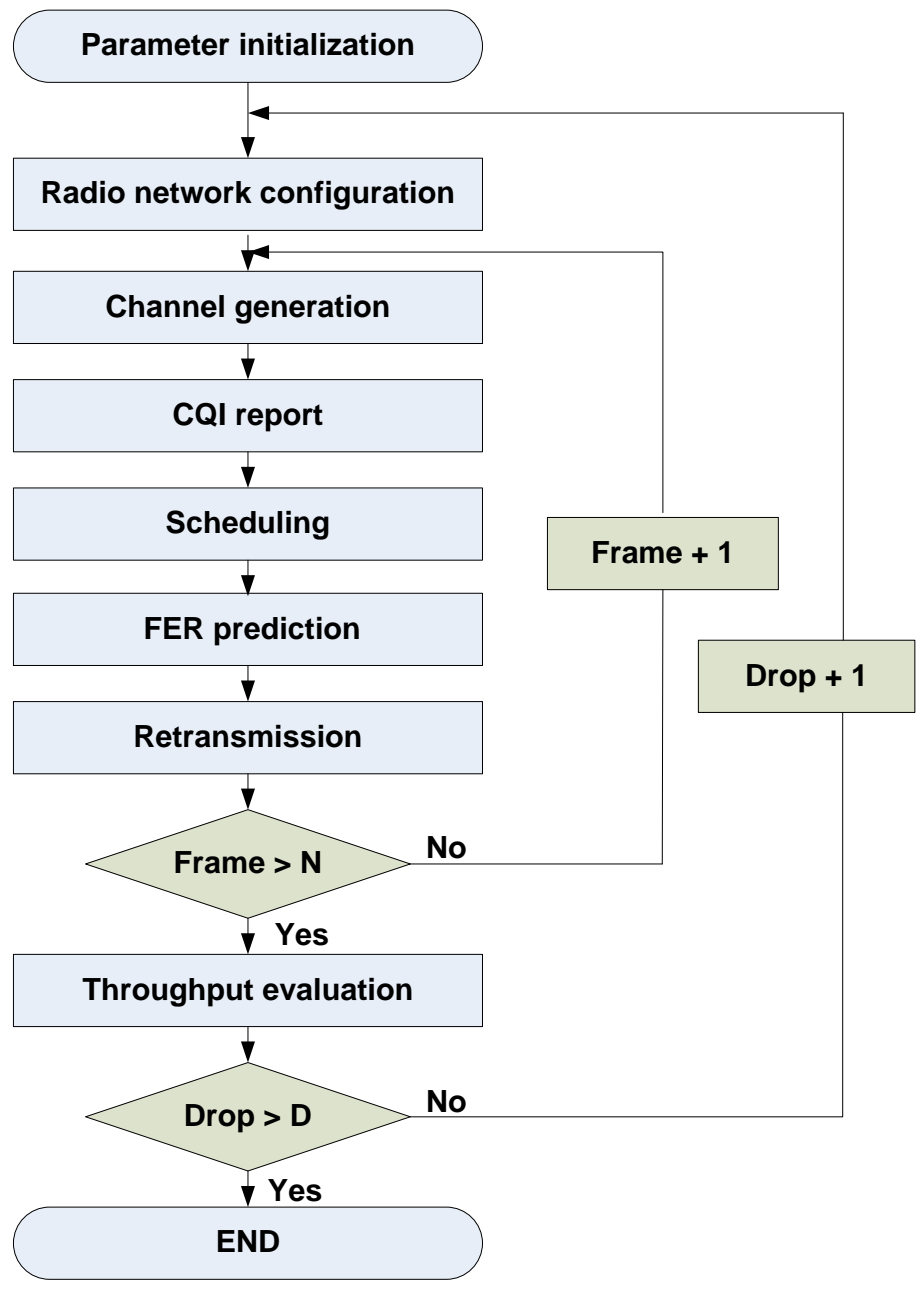

Figure 1. System-level Simulation Procedure

\subsection{Cellular Model}

MSs are uniformly distributed on a two-tier layout of 19 hexagonal cells with three identical sectors in each cell as shown in Figure 2. The path loss for each sector-MS pair is determined by the COST231-Walfisch-Ikegami model, and kept constant during each drop. The path loss is given by

$$
P L[d B]=\left\{\begin{array}{c}
-55.9+38 \times \log _{10}(d)+\left(24.5+1.5 \times f_{c} / 925\right) \times \log _{10}\left(f_{c}\right) \quad N L O S \\
-35.4+26 \times \log _{10}(d)+20 \log _{10}\left(f_{c}\right) \quad \text { LOS }
\end{array}\right.
$$

A shadowing component is generated by a log-normal distributed random variable with zero mean and a standard deviation of $4 \mathrm{~dB}$ for line-of-sight (LOS), and $10 \mathrm{~dB}$ for non-lineof-sight (NLOS). The long-term carrier-to-interference ratio (CIR) is calculated for each sector- 


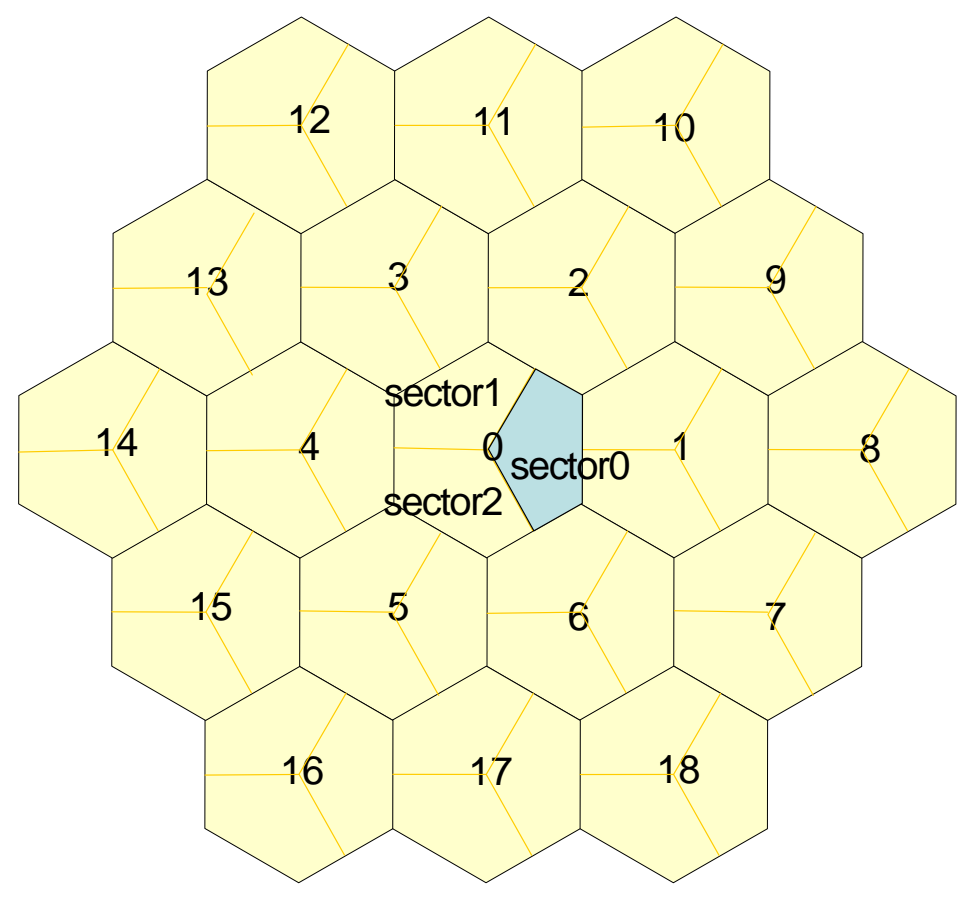

Figure 2. Two-tier Layout of 19 Hexagonal Cells with Three Sectors

MS pair and the sector with the largest CIR are selected as the serving sector by each MS. A multipath profile model and a Doppler frequency are assigned for the channel between each MS and its serving sector and are fixed during a drop.

\subsection{Channel Model}

The spatial channel model (SCM) has been developed to define spatial channel characteristics and a system-level simulation methodology for a single-carrier MIMO system by the ad-hoc group comprised of participants of the 3GPP and 3GPP2 [5]. An extended SCM (ESCM) has also been proposed by Work Package 5 in the European WINNER project [6]. The ESCM supports bandwidths up to $100 \mathrm{MHz}$ in three different outdoor environments. The SCM and ESCM, however, define a single-carrier spatial channel model and thus cannot be directly employed for multi-carrier MIMO systems.

We extend the SCM proposed for a single-carrier MIMO system to that for the MIMOOFDMA system by using discrete Fourier transform (DFT), as shown in Figure 3. For a sector-MS pair, $L$ multipath channel matrices $\left\{\mathrm{H}_{l}\right\}_{l=1, \mathrm{~L}, L}$ with delays $\left\{t_{l}\right\}_{l=1, \mathrm{~L}, L}$ are generated by the SCM. The $(u, s)$ th entry of $\mathrm{H}$, is given as for NLOS

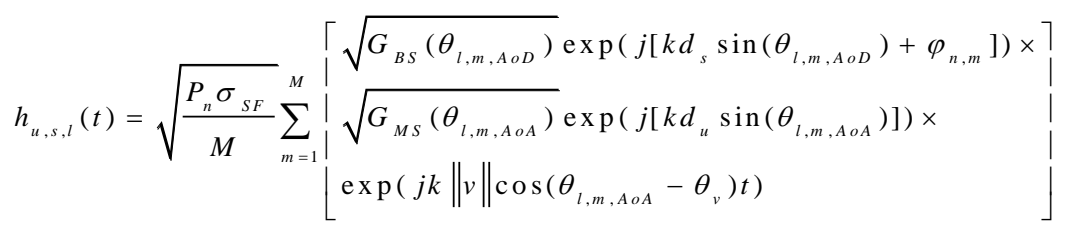

It is also given as for LOS 


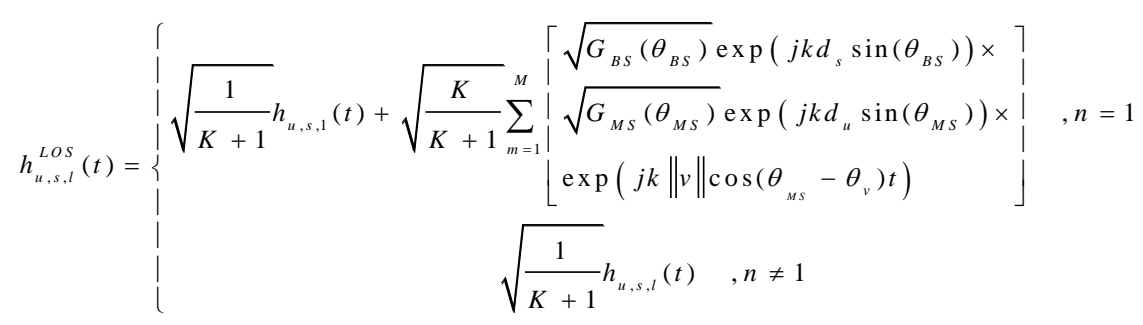

The symbols in (2) and (3) are listed in Table 1.

The channel matrices $\left\{\mathrm{H}_{l}\right\}_{l=1, \mathrm{~L}, L}$ are quantized to $T_{S} / M$ in time, where $T_{S}$ and $M$ denote the interval of an OFDM symbol and the number of sub-carriers, respectively. The quantized $M$ samples $\left\{\hat{\mathrm{H}}_{m}\right\}_{m=1, \mathrm{~L}, M}$ are transformed into the channel matrices of $M$ subcarriers $\left\{\mathrm{H}_{m}\right\}_{m=1, \mathrm{~L}, M}$ by the DFT. The spatial correlation characteristics of a single-carrier MIMO channel are preserved over the DFT process. This extended model for MIMO-OFDM systems is used in [7].
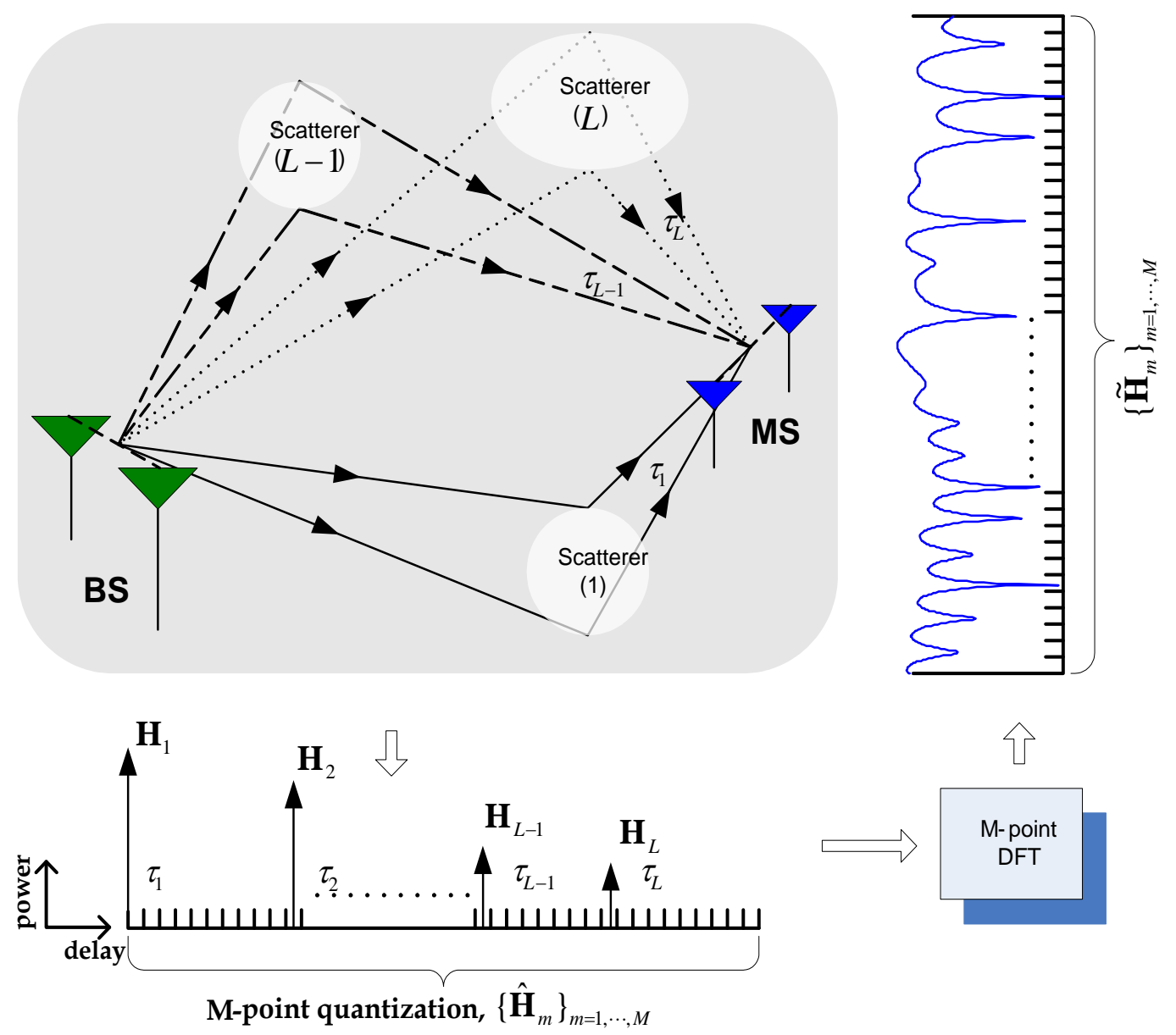
Figure 3. Spatial Channel Model for MIMO-OFDMA System

Table 1. Parameters for Channel Coefficient

\begin{tabular}{|c|c|}
\hline Symbol & Description \\
\hline$P_{n}$ & Power of the $n$th path \\
\hline$\sigma_{S F}$ & Lognormal shadow fading \\
\hline$M$ & Number of subpaths per path \\
\hline$\theta_{n, m, A o D}$ & AOD for the $m$ th subpath of the $n$th path \\
\hline$\theta_{n, m, A O A}$ & AOA for the $m$ th subpath of the $n$th path \\
\hline$\theta_{B S}$ & $\begin{array}{l}\text { Angle of LOS between the BS and MS, with respect to the } \\
\text { broadside of the BS array }\end{array}$ \\
\hline$\theta_{M S}$ & $\begin{array}{l}\text { Angle of LOS between the BS and MS, with respect to the } \\
\text { broadside of the MS array }\end{array}$ \\
\hline$G_{B S}\left(\theta_{n, m, A o D}\right)$ & BS antenna gain of each array element \\
\hline$G_{M S}\left(\theta_{n, m, A O A}\right)$ & MS antenna gain of each array element \\
\hline$k$ & $\begin{array}{c}\text { Wave nmber } 2 \pi / \lambda_{c} \text {, where } \lambda_{c} \text { is the carrier wavelength in } \\
\text { meters. }\end{array}$ \\
\hline$d_{s}$ & $\begin{array}{l}\text { The distance of BS antenna elements from the reference }(\mathrm{s}=1) \\
\text { antenna }\end{array}$ \\
\hline$d_{U}$ & $\begin{array}{l}\text { The distance of MS antenna elements from the reference }(\mathrm{u}=1) \\
\text { antenna }\end{array}$ \\
\hline$\varphi_{n, m}$ & Phase of the $m$ th subpath of the $n$th path \\
\hline$\|\mathbf{v}\|$ & MS velocity vector \\
\hline$\theta_{v}$ & Angle of the MS velocity vector \\
\hline$K$ & Rician $\mathrm{K}$ factor \\
\hline
\end{tabular}

\subsection{SDM and SDMA Scheduling}

During every frame, the serving sector assigns transmission opportunities to active MSs using the channel quality information (CQI) reports per transmit antenna of each sub-band from active MSs. According to the scheduling policy, the multiplexer constructs the transmitted signal vector by combining independent data streams from either a single user or multiple users. For space division multiplexing (SDM), in which individual data streams from a single user are transmitted from different antennas simultaneously, the serving BS selects one MS with the highest scheduling priority per sub-band. On the other hand, space division multiple access (SDMA) schedules multiple data streams from multiple users. For each subband the serving sector chooses the MSs with the highest scheduling priority per transmit antenna. The number of scheduled MSs per sub-band may be as high as the number of 
transmit antennas. The concept of these scheduling policies is illustrated and compared in Figure 4.

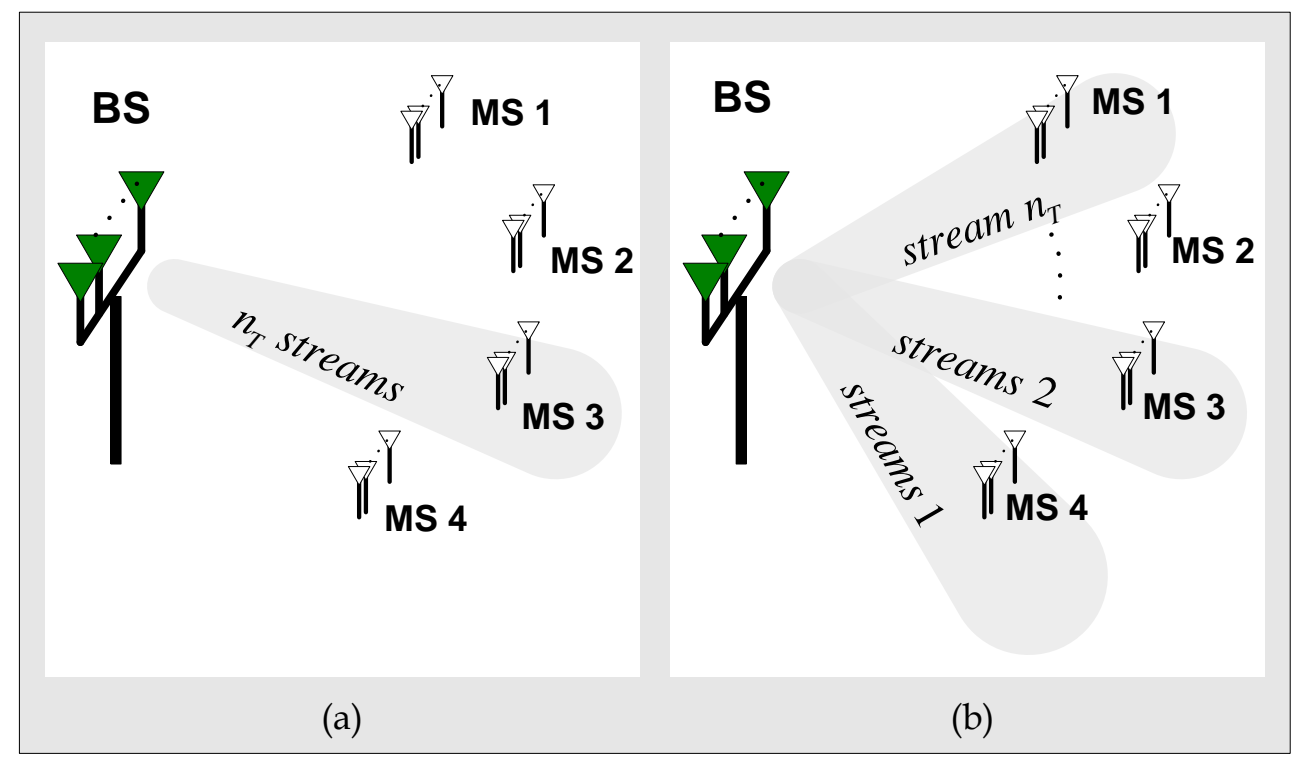

Figure 4. Scheduling Schemes for MIMO-OFDMA: a) SDM scheduling; b) SDMA Scheduling

For SDM scheduling based on proportional fair rule (PFR) the MS scheduled in sub-band $b$ among $K$ active mobiles is given as

$$
k_{b}^{*}=\underset{k \hat{1}\{1,2, \mathrm{~L}, K\}}{\operatorname{argmax}} \frac{\stackrel{\AA^{n_{T}} T_{k, b, t}}{t=1}}{\bar{T}_{k}},
$$

where $\bar{T}_{k}$ is the average transmission rate delivered to MS $k$ in a past window and $T_{k, b, t}$ denotes the instantaneous supported rate for MS $k$ via transmit antenna $t$ in sub-band $b$. All $n_{T}$ data streams transmitted from $n_{T}$ transmit antennas are directed to one MS $k_{b}^{*}$. On the other hand, $n_{T}$ data streams are transmitted to different MSs for the SDMA scheduling. A data stream transmitted via antenna $t$ in sub-band $b$ aims at MS $k_{b, t}^{*}$ and is given by

$$
k_{b, t}^{*}=\underset{k \hat{1}\{1,2, \mathrm{~L}, K\}}{\arg \max } \frac{T_{k, b, t}}{\bar{T}_{k}} .
$$

\subsection{Link to System Interface}

Some methods to predict link performance on the system-level simulation have been proposed. The quasi-static approach was proposed to envisage link performance on the system-level simulation for a CDMA system [8]. An averaged SNR over a packet is calculated and mapped onto the corresponding PER curve to predict the PER. The quasi-static approach provides accurate prediction under static channel conditions. If the channel, 
however, cannot guarantee the static condition, this method can only provide rough estimates. For an OFDMA system, the frequency-selective fading results in unequal SNR across the sub-carriers, this prevents using the quasi-static approach.

The ESM maps a set of signal to inference and noise ratios (SINRs) across sub-carriers and transmit antennas to an equivalent additive white Gaussian noise (AWGN) SNR [9]. The equivalent SNR is used to find an estimate of the PER from the corresponding AWGN reference performance curve. The ESM approach is known to provide reliable link performance for system-level simulation of OFDM [9]. In the following, we introduce a detailed ESM procedure used in this article for evaluation of a MIMO-OFDMA system.

In order to make more accurate prediction of link performance in the system-level simulation of MIMO-OFDMA, the equivalent SNR includes receiver noise, quantization noise, and inter-tone interference (ITI) and coherent loss due to Doppler. The maximum SNR of the receiver is limited by a number of sources such as receiver noise and analog-to-digital quantization noise, which can be approximated as an equivalent additive noise $N_{\max S N R}$. And non-orthogonality of adjacent sub-carriers due to Doppler results in both coherent loss and ITI. The coherent loss term $L_{C}$ and an equivalent additive noise $N_{I T I}$ due to ITI are given as a function of Doppler frequency [9]. The final SINR of the received data stream from transmit antenna $t$ and sub-carrier $m$ is given by

$$
\operatorname{SINR}[m, t]=\frac{L_{C} E_{s}\left|\mathrm{w}(m, t)^{H} \mathrm{~h}(m, t)\right|^{2}}{I_{O S}+I_{O C}+N_{\max S N R}+N_{I T I}+N_{0}},
$$

where $\mathbf{w}(m, t)$ and $\mathrm{h}(m, t)$ denotes an $n_{R}{ }^{\prime} 1$ receiving weight vector and a channel vector transmitted from transmit antenna $t$ and received $n_{R}$ antennas, respectively, and $I_{O S}$ and $I_{O C}$ are the interference from other data streams and other cells, respectively.

An averaged transmission rate achieved by the sub-carriers in a packet is calculated and mapped onto the equivalent SNR $S N R_{\text {equiv }}$. The $S N R_{\text {equiv }}[t]$ per transmit antenna is calculated from the SINRs of the sub-carriers in a packet as follows:

$$
\begin{array}{r}
R_{\text {sum }}[t]=\frac{1}{N_{\text {data }}} \mathrm{a}_{m} \mathrm{C}_{\text {mod }}(\operatorname{SINR}[m, t] / Q) \\
S N R_{\text {equiv }}[t]=Q ? \mathrm{C}_{\bmod }^{-1}\left(R_{\text {sum }}[t]\right),
\end{array}
$$

where $N_{\text {data }}$ is the number of sub-carriers in a packet, $\mathrm{C}_{\mathrm{mod}}(\ngtr$ is the constrained capacity formula for any modulation schemes (e.g. QPSK, 16QAM, or 64QAM), and $Q$ is the gap to the actual AWGN capacity.

The resulting $S N R_{\text {equiv }}$ is mapped to an empirical PER based on the corresponding AWGN reference curve. A set AWGN reference curves are generated a priori for all modulation and coding schemes (MCSs). Finally, a biased coin is flipped for determining whether the packet is in error or not, and a retransmission request is sent in case of packet error.

\section{Capacity Results}


We compare results of system level evaluation of a MIMO-OFDMA system by using the methodology discussed in this article. The 2-elements linear antenna array is assumed at both the transmitter and receiver with the spacing $d_{T}=101$ and $d_{R}=0.51$, respectively. The average sector throughput is obtained by averaging the average transmission rates of drops, each of which is computed by averaging the transmission rates over 4,000 frames for a drop. And the average sector throughput is measured by using a full-buffer traffic model. In the simulation, each MS measures channel quality per transmit antenna and reports the CQIs of the best five bands to its serving sector. Table 2 summarizes some key parameters and values used in the simulation.

We compare the average sector throughput between scheduling policies (e.g., SDM and SDMA) operating in a MIMO-OFDMA system as a function of the number of active MSs in urban micro cellular environments defined in the SCM [5]. For comparison, per-antenna rate control (PARC) [10] and per-common basis rate control (PCBRC) [11] are considered as a transmitter precoding scheme. The PARC transmits independent streams on each transmit antenna, and the data rates on each of the streams are adjusted using the CQI feedback per transmit antenna. For PCBRC, on the other hand, independent streams are precoded by the $n_{T}$ orthonormal weight vectors, which are known at both the receiving and transmitting ends and are fixed for all channel realizations, and the data rates on each of the streams are adjusted using the CQI feedback per transmit weight vector. At both precoding schemes, the total transmit power is uniformly distributed over $n_{T}$ independent data streams. For reference, singleinput single-ouput (SISO) and single-input multiple-output (SIMO) with receive maximum ratio combing (MRC) are also presented.

Figure 5 shows considerable capacity enhancement of both SDM and SDMA over SISO and MRC of received signal. Furthermore, the gain of SDMA over SDM is heightened as the number of MSs Increase, which indicates amplified multi-user diversity order due to SDMA scheduling. Also the result show capacity enhancement of PCBRC over PARC in both SDM and SDMA scheduling. The capacity gap results from transmit beamforming by the common weights, which leads to increase peak transmission rates at a link and as a result increase the system capacity by scheduling the MSs with the higher transmission rates. The capacity gap between PCBRC and PARC shows that a beamforming based precoding scheme can achieve capacity enhancement without additional feedback complexity.

Table 2. Simulation Parameters

\begin{tabular}{c|c}
\hline \hline \multicolumn{1}{c||}{ Parameter } & Value \\
\hline Carrier frequency & $2.3 \mathrm{GHz}$ \\
\hline Bandwidth & $10 \mathrm{MHz}$ \\
\hline Cell structure & 19 (hexagonal grid, 2-ring, 3-sector system) \\
\hline Site-to site distance & Urban micro environments \\
\hline Channel scenario & 2 \\
\hline The num. of BS and MS antennas & COST231-Walfisch-Ikegami model \\
\hline Path-loss model & 0.5 \\
\hline Sector shadowing correlation &
\end{tabular}




\begin{tabular}{c|c}
\hline \multicolumn{1}{c||}{ BS antenna pattern } & $70 \mathrm{deg}(-3 \mathrm{~dB})$ with $20 \mathrm{~dB}$ front-to-back ratio \\
\hline MS antenna gain & $-1 \mathrm{dBi}$ \\
\hline MS noise figure & $7 \mathrm{~dB}$ \\
\hline Thermal noise density & $-174 \mathrm{dBm} / \mathrm{Hz}$ \\
\hline Sector maximum PA power & $20 \mathrm{~W}$ \\
\hline $\begin{array}{c}\text { Overhead channel forward link power } \\
\text { usage }\end{array}$ & $18 \%$ \\
\hline Sector antenna gain with cable loss & $15 \mathrm{~dB}$ \\
\hline Other losses & $10 \mathrm{~dB}$ \\
\hline Maximum CIR achievable & $30 \mathrm{~dB}$ \\
\hline CQI delay & 0 slot (ideal case) \\
\hline Receiver algorithm & Fairness factor $=1.0$ \\
\hline PF Scheduler & 1024 \\
\hline The num. of total sub-carriers & 768 \\
\hline The num. of data sub-carriers & 24 \\
\hline The num. of bands for a frame & 3 \\
\hline The num. of max. retransmission & 3686 bits \\
\hline Forward link encoder packet sizes & $192,384,576,768,1152,1536,2304,3072$, \\
\hline \hline
\end{tabular}

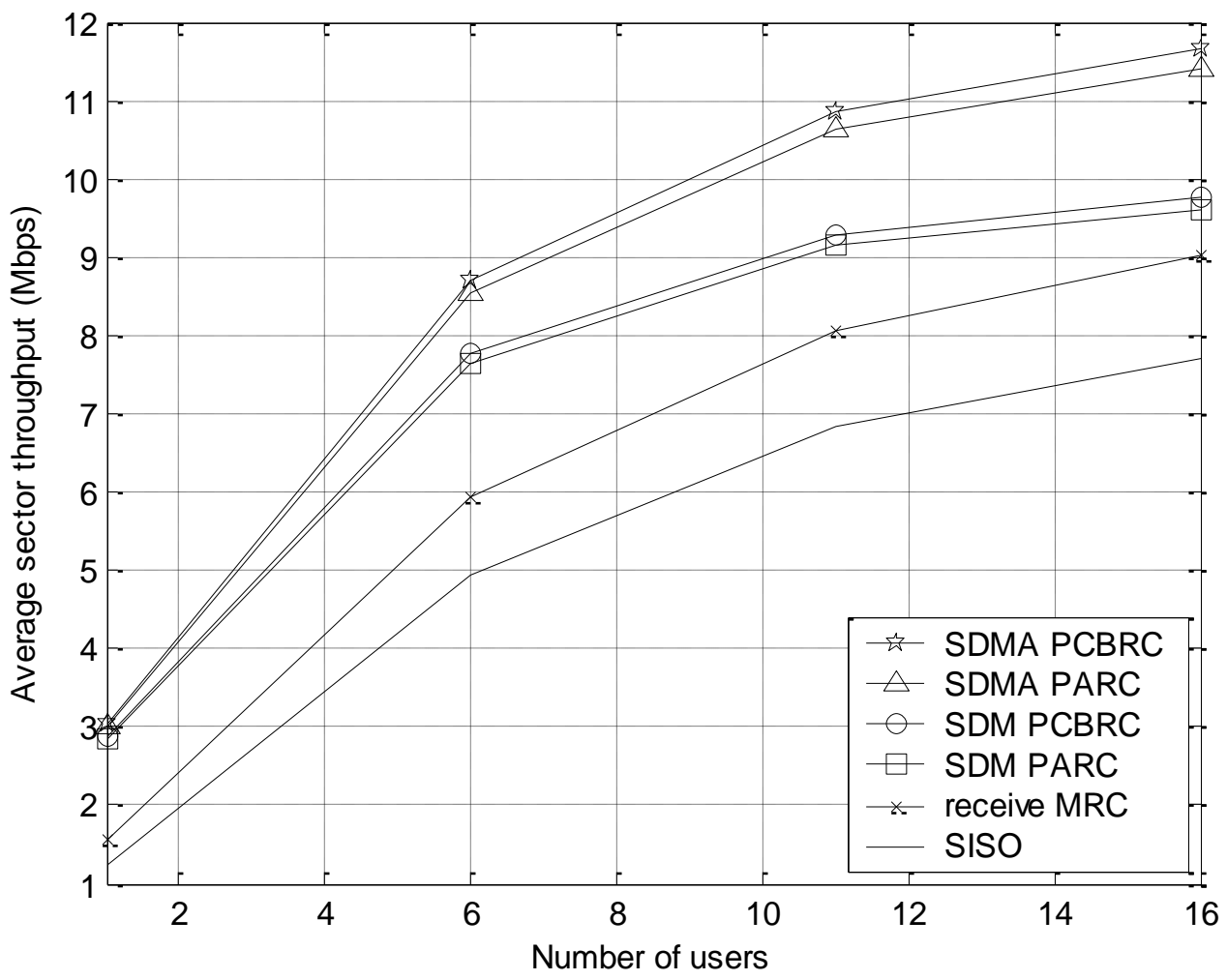

Figure 5. The Average Sector Throughput of MIMO-OFDMA in Urban Micro Environments 


\section{Conclusion}

We discuss a system-level methodology for the purpose of evaluating MIMOOFDMA system and present the system-level evaluation results. Results show the interaction between multi-user scheduling and MIMO schemes. The system-level capacity enhancement due to multi-user scheduling is achieved by increasing the capacity variance at each link, which is realized by incorporating both SDMA scheduling and beamforming-based precoding schemes into OFDMA.

\section{Acknowledgements}

This research was supported by the MSIP(Ministry of Science, ICT and Future Planning), Korea, under the Global IT Talent support program (NIPA-2014ITAH0904140110020001000100100) supervised by the NIPA(National IT Industry Promotion Agency), and also it was supported by the Basic Science Research Program through the National Research Foundation of Korea (NRF) funded by the Ministry of Education, Science and Technology (2013R1A1A1005731)

\section{References}

[1]. 3GPP TSG RAN WG1, "Text Proposal on Downlink and Uplink Transmission Schemes for E-UTRA", R1-051577, Seoul, Korea, (2005) November.

[2]. H. S. Jo, "Spectrum Sharing SDMA with Limited Feedback", Throughput Analysis, KSII Trans. on Internet and Information Systems, vol. 6, no. 12, (2012) December, pp. 3237-3256.

[3]. C. Mun and H. S. Jo, "Throughput Analysis of Transmit-Nulling SDMA with Limited Feedback", EURASIP Journal on Wireless Communications and Networking (2013) November, pp. 2013:270.

[4]. H. S. Jo and C. Mun, "Transmit-nulling SDMA for coexistence with fixed wireless service", Journal of Electromagnetic Engineering and Science, vol. 11, (2011), pp. 34-41.

[5]. "3GPP-3GPP2 Spatial Channel Model Ad-Hoc Group”, Spatial Channel Model Text, in SCM-134, (2003) April 22.

[6]. D. S. Baum, J. Hansen, and J. Salo, “An Interim Channel Model for Beyond-3G Systems”, Extending the 3GPP SCM, in Proc. of IEEE VTC Spring, (2005), pp. 3132-3136.

[7]. H. S. Jo, "Codebook-based precoding for SDMA-OFDMA with spectrum sharing," ETRI Journal, vol. 33, no. 6, (2011) November, pp. 831-840.

[8]. R. Ratasuk, A. Ghosh, and B. Classon, "Quasi-Static Method for Prediction Link-Level Performance, in Proc. of IEEE VTC", (2002), pp. 1298-1302.

[9]. Qualcomm, "Simulation Assumptions for Link Error Prediction Based on Equivalent SNR for Enhanced Broadcast-Multicast Physical Layer", C30-20040607-061, (2004) June.

[10]. Lucent, "Increasing MIMO throughput with per antenna rate control", TSG_R WG1 document TSGR1, vol. 0879, no. 01, (2001) August.

[11]. D. Y. Kim, M. W. Lee, C. Mun, J. K. Han, and J. G. Yook, "System Level Performance Evaluation of Spatial Multiplexing with Per Common Basis Rate Control, in Proc.”, IEEE VTC Fall, (2005), pp. 2463-2467.

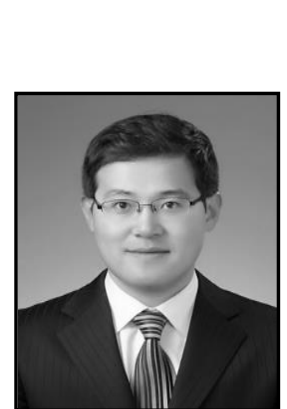

\section{Author}

Han-Shin Jo received the B.S., M.S., and Ph.D. degrees in electrical and electronics engineering from Yonsei University, Seoul, Korea, in 2001, 2004, and 2009, respectively. He is an Assistant Professor with the Department of Electronics and Control Engineering, Hanbat National University in Korea. He was a Postdoctoral Research Fellow in Wireless Networking and Communications Group, the Department of Electrical and Computer Engineering, the University of Texas at Austin from 2009- 
International Journal of Multimedia and Ubiquitous Engineering

Vol. 10, No. 2 (2015)

11. He developed Long Term Evolution (LTE) base station in Samsung Eletronics in 2011-12. Dr. Jo received Samsung Electronics Graduate Fellowship in 2006-08, Korea Research Foundation BrainKorea21 Graduate Fellowship in 2006-07, Korea Research Foundation Postdoctoral Fellowship in 2009, and 2011 ETRI Journal Best Paper Award. His research interests include small cells, heterogeneous network, wireless ad-hoc network, stochastic geometry, and wireless broadband transmission. 